This is the accepted manuscript made available via CHORUS. The article has been published as:

Discovery of highly spin-polarized conducting surface states in the strong spin-orbit coupling semiconductor $\mathrm{Sb}\{2\} \mathrm{Se}_{-}\{3\}$

Shekhar Das, Anshu Sirohi, Gaurav Kumar Gupta, Suman Kamboj, Aastha Vasdev, Sirshendu Gayen, Prasenjit Guptasarma, Tanmoy Das, and Goutam Sheet Phys. Rev. B 97, 235306 - Published 8 June 2018 DOI: 10.1103/PhysRevB.97.235306 


\title{
Discovery of highly spin-polarized conducting surface states in the strong spin-orbit coupling semiconductor $\mathrm{Sb}_{2} \mathrm{Se}_{3}$
}

\author{
Shekhar Das ${ }^{1}$, Anshu Sirohi ${ }^{1}$, Gaurav Kumar Gupta ${ }^{2}$, Suman Kamboj ${ }^{1}$, Aastha \\ Vasdev $^{1}$, Sirshendu Gayen ${ }^{1}$, Prasenjit Guptasarma ${ }^{3}$, Tanmoy Das ${ }^{2}$, and Goutam Sheet ${ }^{1 *}$ \\ ${ }^{1}$ Department of Physical Sciences, Indian Institute of Science Education and Research Mohali, \\ Sector 81, S. A. S. Nagar, Manauli, PO: 140306, India \\ ${ }^{2}$ Department of Physics, Indian Institute of Science, Bangalore 560012, India and \\ ${ }^{3}$ Department of Physics, University of Wisconsin, Milwaukee, Wisconsin 53211, USA
}

\begin{abstract}
Majority of the $\mathrm{A}_{2} \mathrm{~B}_{3}$ type chalcogenide systems with strong spin-orbit coupling (SOC), like $\mathrm{Bi}_{2} \mathrm{Se}_{3}, \mathrm{Bi}_{2} \mathrm{Te}_{3}$ and $\mathrm{Sb}_{2} \mathrm{Te}_{3}$ etc., are topological insulators. One important exception is $\mathrm{Sb}_{2} \mathrm{Se}_{3}$, where a topological non-trivial phase was argued to be possible under ambient conditions, but such a phase could be detected to exist only under pressure. In this paper, we show that $\mathrm{Sb}_{2} \mathrm{Se}_{3}$, like $\mathrm{Bi}_{2} \mathrm{Se}_{3}$ displays generation of highly spin-polarized current under mesoscopic superconducting point contacts as measured by point contact Andreev reflection spectroscopy. In addition, we observe a large negative and anisotropic magnetoresistance of the mesoscopic metallic point-contacts formed on $\mathrm{Sb}_{2} \mathrm{Se}_{3}$. Our band structure calculations confirm the trivial nature of $\mathrm{Sb}_{2} \mathrm{Se}_{3}$ crystals and also reveal two trivial surface states one of which shows large spin-splitting due to Rashba type SOC. The observed high spin polarization and related phenomena in $\mathrm{Sb}_{2} \mathrm{Se}_{3}$ can be attributed to this spin-splitting.
\end{abstract}

Within the band theory of solids metals and insulators are distinguished based on a band gap which is either zero for metals or non-zero for insulators. A topological insulator behaves like an insulator with a band gap in the bulk but the surface contains gapless conducting states protected by time reversal symmetry ${ }^{1-4}$. In such systems strong spin-orbit coupling acts as an effective magnetic field pointing in a spin dependent direction thereby giving rise to non-zero spin-polarization of the conducting surface states ${ }^{5-7}$. In other words, the charge carriers corresponding to these surface states have the spin angular momentum locked with the orbital angular momentum which means carriers with definite momentum direction have definite spin. The spin polarization of the surface states of topological insulators were measured in the past by a number of techniques. The most widely exploited techniques included spin resolved ARPES ${ }^{8-10}$ and using circularly polarized photons to excite spinpolarized photo current ${ }^{11-13}$. Electrical methods based on fabrication of devices involving topological insulators have also been employed ${ }^{14,15}$. More recently it was shown that point contact Andreev reflection spectroscopy using a sharp tip of a conventional superconductor on the surface of a topological insulator can also be used to measure the spin polarization of the surface states through the mesurement of the degree of supression of Andreev reflection ${ }^{16}$.

Andreev reflection at an interface between a conventional superconductor and a topological material should be analyzed carefully as coupling between the superconducting order and the topological phase may be complex, particularly because of the possibility of the emergence of a topological superconductor at such interfaces ${ }^{17-22}$.

${ }^{*}$ Electronic address: goutam@iisermohali.ac.in
This aspect was studied in the past and it was found that the proximity induced superconductivity in point contact geometries on topological insulators have a far greater non-topological character than topological ${ }^{23}$. From Andreev reflection experiments on various topological insulators it was found that spin polarization in doped topological insulators can vary with the level of doping. The topological insulators $\mathrm{Bi}_{2} \mathrm{Te}_{3}$ and $\mathrm{Sb}_{2} \mathrm{Te}_{3}$ showed spin polarization of $70 \%$ and $57 \%$ respectively ${ }^{16}$. In all these cases, a conventional modified Blonder-TinkhamKlapwijk (BTK) theory was used for the analysis ${ }^{24,25}$.

Based on earlier band structure calculations ${ }^{26,27}$ $\mathrm{Sb}_{2} \mathrm{Se}_{3}$, a member of the $\mathrm{A}_{2} \mathrm{~B}_{3}$ type chalcogenide family with high spin orbit coupling was categorized as a trivial band insulator under ambient conditions. Some experiments indicated the possibility of a topologically nontrivial character emerging in $\mathrm{Sb}_{2} \mathrm{Se}_{3}$ under a pressure of several giga pascals $(\mathrm{GPa})^{28-31}$. More recent band structure calculations claimed $\mathrm{Sb}_{2} \mathrm{Se}_{3}$ in fact, can be a topological insulator under ambient conditions ${ }^{32}$. In this Letter, from spin-polarized Andreev reflection spectroscopy measurements, we show that the surface of $\mathrm{Sb}_{2} \mathrm{Se}_{3}$ contains highly spin polarized surface states (with up to $70 \%$ spin polarization) that take part in conduction leading to the generation of highly spin-polarized current. The observations are consistent with our band structure calculations which reveal the existence of two trivial surface states in $\mathrm{Sb}_{2} \mathrm{Se}_{3}$ one of which undergoes Rashba type SOC induced spin-splitting.

First we confirmed the surface quality of the $\mathrm{Sb}_{2} \mathrm{Se}_{3}$ crystals using a low-temperature and ultra high vacuum scanning tunneling microscope working down to $300 \mathrm{mK}$. The crystals were cleaved in - situ at $80 \mathrm{~K}$ under ultrahigh vacuum and were immediately transferred to the STM measurement head kept at low temperature. A large area STM image of the $\mathrm{Sb}_{2} \mathrm{Se}_{3}$ surface shows multiple extended atomic terraces with sharp steps as shown in 
(a)

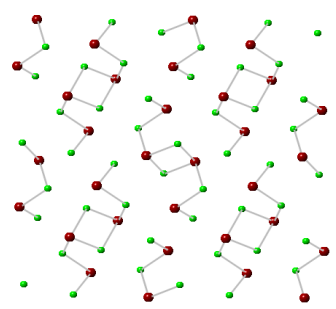

(c)

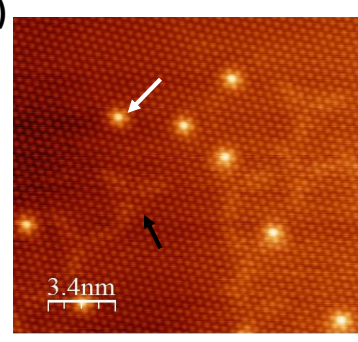

(b)

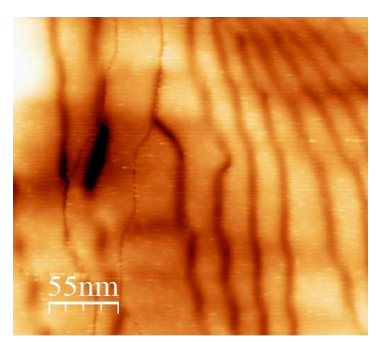

(d)

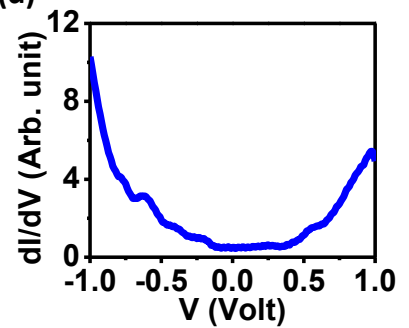

Figure 1: (a) Crystal structure of $\mathrm{Sb}_{2} \mathrm{Se}_{3}$ viewed from the [001] direction of the crystal. (b) A large area $(281 \mathrm{~nm} \mathrm{x}$ $281 \mathrm{~nm}$ ) STM topograph on $\mathrm{Sb}_{2} \mathrm{Se}_{3}$ showing atomically sharp terraces. The parameters are $\mathrm{T}=17 \mathrm{~K}, \mathrm{~V}_{s}=700 \mathrm{mV}$ and $\mathrm{I}_{s}=100 \mathrm{pA}$. (c) A representative atomic resolution image over an area of $17 \mathrm{~nm} \times 17 \mathrm{~nm}$ on one of the terraces. (d) A scanning tunneling conductance spectrum recorded away from the defects.

Figure 1(b). Small area scans on top of the terraces and inside the trenches between two atomic terraces resolved atoms and the defect states (Figure 1(c)). Two types of defects are observed with one type having triangular shapes (black arrow) and the other type appear as bright spots (white arrow). The respective sides of the triangles for different triangular-shaped defects are all parallel to each other and all such defects are randomly distributed throughout the crystal surface. The triangular defects are known to be associated with Se-vacancy in the binary selenide family of materials like $\mathrm{Bi}_{2} \mathrm{Se}_{3}{ }^{33,34}$. The bright spots observed here might be due to $\mathrm{Sb}$ defects in the crystals. In figure $1(\mathrm{~d})$ we plot a typical local density of states (LDOS) spectrum recorded on $\mathrm{Sb}_{2} \mathrm{Se}_{3}$, away from the defects. The LDOS spectrum exhibits a "U"-shape with a flat bottom indicating a gap of $\sim 1 \mathrm{eV}$ opening with the Fermi energy falling within the gap. The LDOS within the gap region does not become absolutely zero. These low-energy states could emerge from possible surface states in $\mathrm{Sb}_{2} \mathrm{Se}_{3}$. However, no clear signature of a surface "Dirac cone" was observed which is again consistent with the non-topological nature of $\mathrm{Sb}_{2} \mathrm{Se}_{3}$.

Point-contact Andreev reflection spectroscopy measurements ${ }^{35-37}$ on $\mathrm{Sb}_{2} \mathrm{Se}_{3}$ crystals were performed using sharp tips of two conventional superconductors $\mathrm{Pb}$ and $\mathrm{Nb}$. In Figure 2(a,b,c) and Figure 2(d,e) we show the representative Andreev reflection spectra obtained on $\mathrm{Sb}_{2} \mathrm{Se}_{3}$ with the $\mathrm{Pb}$ and the $\mathrm{Nb}$ tips respectively. The sharp dip structure at $V=0$ along with two shallow

peaks symmetric about $V=0$ in the normalized $d I / d V$ spectra indicate considerable suppression of Andreev reflection. The black lines show the fit to the experimentally obtained spectra using Blonder-Tinkham-Klapwijk (BTK) theory modified for the finite spin-polarization of the non-superconducting electrode. The extracted values of spin-polarization $P$ are also shown. For low values of $Z$, the spin polarization is measured to be almost $70 \%$. For both $\mathrm{Nb}$ and $\mathrm{Pb}$ tips the extracted values of $P$ is also seen to slightly depend (linearly) on $Z$ as shown in Figure 2(f). The solid lines in Figure 2(f) show linear extrapolation of the $Z$-dependence of $P$ to $Z=0$. This is the expected intrinsic value of the spin-polarization (for $Z=0$ ). The intrinsic spin-polarization in this case is found to be approximately $65 \%$ which is significantly large compared to some of the strong elemental ferromagnetic metals ${ }^{38}$ like Fe $(P=40 \%)$, Co $(P=42 \%)$ and Nickel $(P=39 \%)$ and is comparable to the spin polarization of $70 \%$ measured by Andreev reflection spectroscopy in $\mathrm{Bi}_{2} \mathrm{Te}_{3}{ }^{16}$. It is interesting to note that unlike in case of $\mathrm{Bi}_{2} \mathrm{Te}_{3}$, where two gap amplitudes were considered for fitting the Andreev reflection spectra, in case of $\mathrm{Sb}_{2} \mathrm{Se}_{3}$, only a single gap amplitude $(\Delta)$ was required which varied between $1.3 \mathrm{meV}$ and 1.5 $\mathrm{meV}$, as expected for $\mathrm{Nb}$ point contacts on a regular metal. For $\mathrm{Pb}$ point contacts, $\Delta$ remained comparable to the bulk gap of $\mathrm{Pb} \sim 1 \mathrm{meV}$. Thus, the analysis involved only three freely varying fitting parameters $P, Z$ and $\Gamma$, the effective broadening parameter. The observation of high value of spin polarization in $\mathrm{Sb}_{2} \mathrm{Se}_{3}$ indicates that though the system is not categorized as a topologically nontrivial system, there might be trivial surface states with non-trivial spin-texture present due to the strong spin-orbit coupling. It should be noted that this observation is not related to the pressure induced topological phase that was earlier observed on $\mathrm{Sb}_{2} \mathrm{Se}_{3}$ because the high spin polarization is observed even with soft tips made of superconducting $\mathrm{Pb}$ which cannot withstand a pressure of the order of several tens of GPa that is required to induce such a phase. Furthermore, it is known that a superconducting phase of $\mathrm{Sb}_{2} \mathrm{Se}_{3}$ is realized under high pressure ${ }^{39}$, but we did not observe any signature of superconductivity of $\mathrm{Sb}_{2} \mathrm{Se}_{3}$.

In the context of certain other topological materials where a surface spin polarization was expected (as in case of metallic point contacts on $\mathrm{TaAs})^{20}$, it was observed that the point contact resistance showed anisotropy when the magnetic field was rotated in a plane perpendicular to the direction of the injected current. In order to investigate that possibility in $\mathrm{Sb}_{2} \mathrm{Se}_{3}$, we have performed similar experiments on the point contacts formed on $\mathrm{Sb}_{2} \mathrm{Se}_{3}$ at $V=0$. As shown in Figure 3 (a) for point contacts with $\mathrm{Nb}$ and Figure 3(b) for point contacts with $\mathrm{Pb}$, the magnetoresistance of the point-contacts on $\mathrm{Sb}_{2} \mathrm{Se}_{3}$ are highly anisotropic. For $\mathrm{Nb}$ point contacts the anisotropy is well described by a $\cos 2 \phi$ field-angle dependence, where $\phi$ represents the relative angle made by the magnetic field vector with respect to the axis $a$ in the basal plane. For 

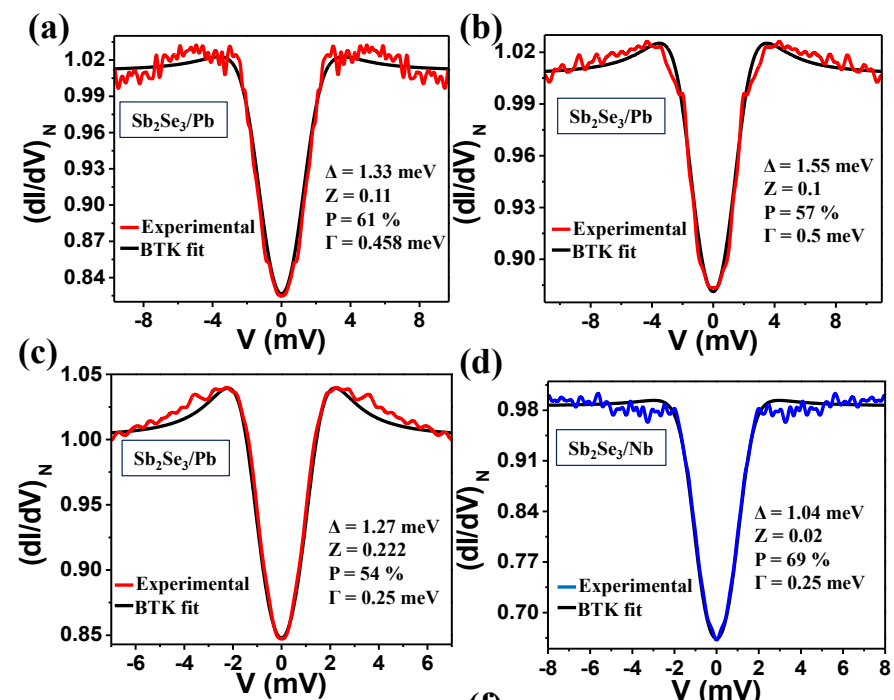

(d)
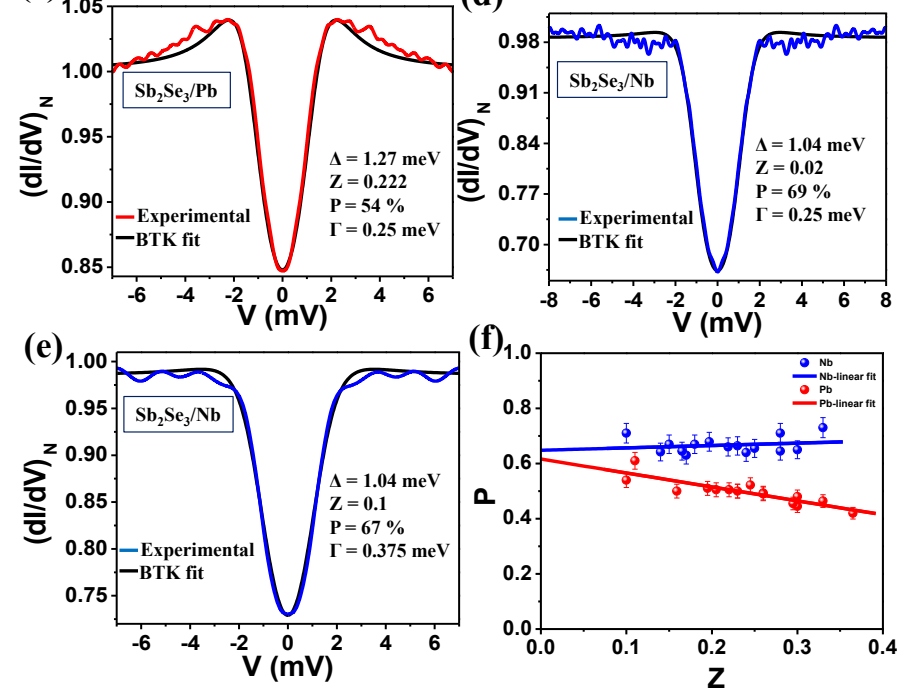

Figure 2: Normalized $d I / d V$ spectra for point-contacts on $\mathrm{Sb}_{2} \mathrm{Se}_{3}$ with $(\mathrm{a}, \mathrm{b}, \mathrm{c}) \mathrm{Pb}$ tip and (d, e) $\mathrm{Nb}$ tip. The black lines show BTK fits with spin-polarization included. (f) Spinpolarization $(\mathrm{P})$ vs. barrier strength $(\mathrm{Z})$ plot. The solid lines show extrapolation to $Z=0$ where the spin-polarization approaches $70 \%$.

$\mathrm{Pb}$ point contacts, the anisotropy deviated slightly from a $\cos 2 \phi$ dependence and it fitted well with $\cos (2.25) \phi$. The approximate $\cos 2 \phi$ dependence observed here is similar to what was observed in case of TaAs point contacts in the past ${ }^{20}$. Furthermore, a $\cos 2 \phi$ angular dependence of the anisotropic magnetoresistance is routinely observed in certain systems with large spin polarization ${ }^{40}$ including the conventional ferromagnets ${ }^{41}$. Therefore, the observed field-angle dependence here further supports the existence of magnetic correlations and the occurence of a spin-polrized transport through the point contacts on $\mathrm{Sb}_{2} \mathrm{Se}_{3}$.

It is also important to investigate whether the measured spin polarization emerges only under point contacts as it seems to be the case in $\mathrm{TaAs}^{42}$ or if that is an intrinsic surface property. In order to confirm that we measured the field-angle dependence of resistance of the crystal in a conventional four-probe geometry. The fourprobe resistance is also seen to be anisotropic confirming that the anisotropic magnetoresistance is not confined to the mesoscopic point-contacts, but originates from magnetic correlations on $\mathrm{Sb}_{2} \mathrm{Se}_{3}$ surface. As it can be seen in Figure 3(c), the anisotropy in magnetoresistance is
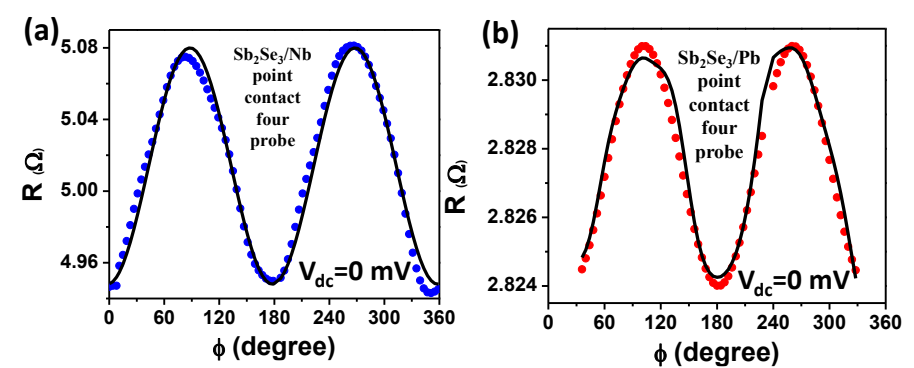

(c)
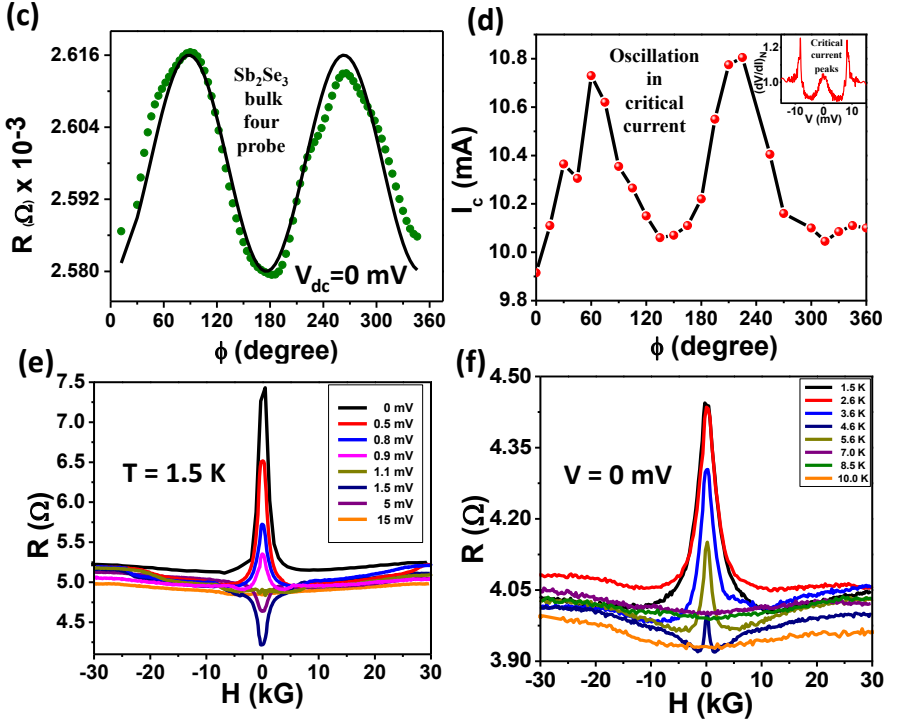

Figure 3: Anisotropic magnetoresistance of (a) a pointcontact on $\mathrm{Sb}_{2} \mathrm{Se}_{3}$ with $\mathrm{Nb}$. The black line shows a $\cos 2 \phi$ fit. (b) a point-contact with $\mathrm{Pb}$. The black line shows a $\cos (2.25) \phi$ fit. (c) bulk $\mathrm{Sb}_{2} \mathrm{Se}_{3}$ crystal. The black line shows a $\cos 2 \phi$ fit (d) Anisotropy of the critical current of a superconducting point-contact in the thermal regime. The corresponding $d V / d I$ spectrum is shown in the inset. (e) Magnetoresistance of a $\mathrm{Pb} / \mathrm{Sb}_{2} \mathrm{Se}_{3}$ point contact at different bias. $\mathrm{T}=$ $1.5 \mathrm{~K}$. (f) Temperature dependence of the magnetoresistance.

1000 times smaller than that in the point contacts; but, again shows a reasonably good $\cos 2 \phi$ dependence. The anisotropy of the magnetoresistance of the mesoscopic point-contacts and the four probe surface resistance can be attributed to the possible anisotropy of the effective spin-orbit coupling in $\mathrm{Sb}_{2} \mathrm{Se}_{3}$. Such anisotropy of the four-probe magnetoresistance was earlier observed in the topological insulator $\mathrm{Bi}_{2} \mathrm{Se}_{3}{ }^{43}$. Therefore, the observed anisotropy also indicates that the highly spin-polarized surface states are indeed governed by the strong spinorbit coupling in $\mathrm{Sb}_{2} \mathrm{Se}_{3}$.

When the superconducting electrodes are in proximity of highly spin-polarized states, the critical current of the point contacts must also be modulated by the magnetic properties of such states. For investigating the modulation of critical current with magnetic field-angle, we first established point-contacts away from the ballistic regime such that Maxwell's contribution to the total point-contact resistance becomes significant and conduc- 

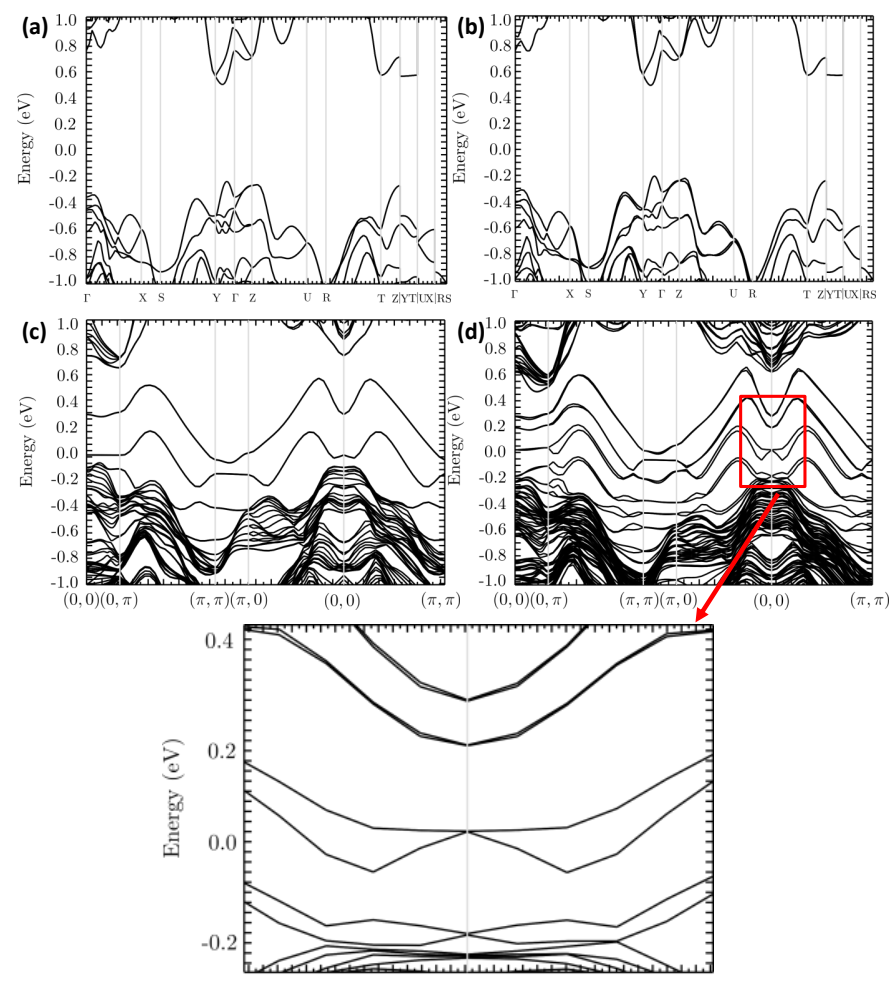

$(0,0)$

Figure 4: Band structure of $\mathrm{Sb}_{2} \mathrm{Se}_{3}$ (a) bulk, without SOC, (b) bulk, with SOC, (c) for finite lattice, without SOC and (d) for finite lattice, with SOC.

tance $\operatorname{dips}^{37}$ (peaks in $d V / d I$ ) associated with critical current becomes prominent. Such a spectrum is shown in the inset of Figure 3(d). The position of the conductance dip provides a direct measure of the critical current. After that we rotated the magnetic field in the basal plane of the crystal and recorded the spectrum for different directions of the applied magnetic field. As shown in Figure 3(d), the critical current also oscillates with the direction of the applied magnetic field in striking agreement with the anisotropic magnetoresistance presented in Figure 3(a,b,c).

In the context of the spin polarized transport in $\mathrm{Sb}_{2} \mathrm{Se}_{3}$ single crystals, it should also be noted that the pointcontacts obtained in the ballistic regime of transport also show negative magnetoresistance. The negative magnetoresistance data is shown in Figure $3(\mathrm{f})$. The black curve shows the magnetoresistance at $1.5 \mathrm{~K}$. The resistance shows a peak at zero magnetic field which is suppressed by a weak magnetic field. As the temperature is increased, the zero-field peak is systematically suppressed. This effect is completely suppressed at $7 \mathrm{~K}$. In order to confirm whether the magnetoresistance is due to the superconductivity of $\mathrm{Pb}$ alone, the experiment was carried out at different bias applied across the tip and sample (Figure 3(e)). It is seen that with increasing the bias the peak at zero field gradually smears out and, surprisingly, becomes a dip at a bias of $1.5 \mathrm{mV}$. With further increasing the bias, the dip starts fading away and the magnetoresistance disappears at a bias of $15 \mathrm{mV}$ which is an order of magnitude higher than the bulk superconducting gap of $\mathrm{Pb}$. This shows that the magnetoresistance is not entirely due to the superconductivity of $\mathrm{Pb}$, but $\mathrm{Sb}_{2} \mathrm{Se}_{3}$ surface also has non-trivial magnetic correlations. In fact, such negative magnetoresistance and a reversal of that with an applied gate bias was observed in the past in certain topologically non-trivial systems (and systems hosting Dirac electrons) where the reversal of magnetization was attributed to a transition from weak anti-localization dominated transport to weak localization dominated transport ${ }^{44,45}$. While such a possibility cannot be ruled out in our point contact data on $\mathrm{Sb}_{2} \mathrm{Se}_{3}$, proving the existence of the same in a point contact geometry is non-trivial and may involve measurements on nano-patterned thin flakes of the crystals with a gate bias, which is beyond the scope of this work.

Based on the key experimental observations highlighted above, it is now imperative to understand the possibility of spin polarized surface states by investigating the band structure of our $\mathrm{Sb}_{2} \mathrm{Se}_{3}$ crystals and the role of spin orbit coupling. Our $\mathrm{Sb}_{2} \mathrm{Se}_{3}$ crystals have an orthorhombic structure with space group Pnma. Experimentally determined lattice constants are $a=4.0345 \AA$, $\mathrm{b}=11.5681 \AA, \mathrm{c}=12.7341 \AA ; \alpha=\beta=\gamma=90^{\circ}$. Band structure calculations were performed using density functional theory within the Local Density Approximation (LDA) exchange correlation as implemented in Vienna ab-initio simulation package (VASP) ${ }^{46}$. Projected augmentedwave (PAW) pseudo-potentials are used to describe the core electron in the calculation ${ }^{47}$. The electronic wavefunction is expanded using plane wave up to a cutoff energy of $265 \mathrm{eV}$. Brillouin zone sampling is done by using a (10x10x10) Monkhorst-Pack k-grid. Both atomic position and cell parameters are allowed to relax, until the forces on each atom are less than $0.01 \mathrm{eV} / \AA$.

$\mathrm{Sb}_{2} \mathrm{Se}_{3}$ in the typical rhombohedral structure does not have sufficient SOC strength to cause topological band inversion $^{2}$. With sufficient van-der Waal interaction, a DFT calculation however predicted that $\mathrm{Sb}_{2} \mathrm{Se}_{3}$ could become a topological insulator, without any experimental verifications to date ${ }^{32}$. The presently studied orthorhombic phase of $\mathrm{Sb}_{2} \mathrm{Se}_{3}$ (our crystals) has higher crystal symmetry, which has the tendency to reduce the SOC strength in the bulk, and hence is even less suitable for the topological insulating phase ${ }^{48}$. Indeed, our DFT calculations for the bulk phase indicated no signature of band inversion with SOC. In Figure (4) we show our computed DFT band structure without and with SOC for bulk as well as for finite size system. Without SOC, the material is a band insulator with gap $\sim 1 \mathrm{eV}$, compare Figures 4(a) and 4(b), much larger than the band gap of $\mathrm{Sb}_{2} \mathrm{Se}_{3}$ in the rhombohedral phase shown in Figure 4(a). Therefore, SOC fails to switch this band ordering.

However, interestingly, we found two trivial surface states without SOC for the cleave perpendicular to the c-axis (see structure in Figure 1(a)). This is the cleaved 
plane we have probed in PCAR experiments. Among these two states, one shows a large splitting at the surface due to Rashba-type SOC. The spin-splitting is about $0.8 \mathrm{eV}$ shown in Figure 4(d), which is comparable to the experimental value. Our DFT calculations also yield a tiny magnetic phase which is however too small to open any appreciable band gap at the time-reversal invariant $\Gamma$ point. While there is a possibility that the small anisotropic field-angle dependence of magnetoresistance (Figure 3) could originate from this tiny magnetic phase, additional experiments are required to confirm that. Therefore, DFT calculations support our observation of large spin-orbit splitting at the surface of $\mathrm{Sb}_{2} \mathrm{Se}_{3}$ which is not protected by bulk topology, but with a time-reversal symmetry as in the case of a quintessential Rashba-SOC split quantum gases.

In conclusion, we have employed spin-polarized Andreev reflection spectroscopy and detected highly spinpolarized surface states in the topologically trivial band insulator $\mathrm{Sb}_{2} \mathrm{Se}_{3}$ with strong spin-orbit coupling. Fur- thermore, we observed highly anisotropic magnetoresistance in the basal plane of the $\mathrm{Sb}_{2} \mathrm{Se}_{3}$ crystals indicating the existence of magnetic correlations. All our experimental observations indicate that $\mathrm{Sb}_{2} \mathrm{Se}_{3}$ is a topologically trivial system. This is again consistent with our band structure calculations which revealed the existence of trivial surface states in $\mathrm{Sb}_{2} \mathrm{Se}_{3}$ one of which undergoes spin splitting due to Rashba-type SOC thereby giving rise to the observed large value of the spin polarization and the related magnetic correlations.

We acknowledge fruitful discussions with Umesh Waghmare. PG acknowledges support from an AFOSRMURI grant. GS acknowledges financial support from the research grants of (a) Swarnajayanti fellowship awarded by the Department of Science and Technology (DST), Govt. of India under the grant number DST/SJF/PSA-01/2015-16, and (b) the research grant from DST-Nanomission under the grant number SR/NM/NS-1249/2013.
[1] L. Fu, C. L. Kane, and E. Mele, J. Phys. Rev. Lett. 98, 106803 (2007).

[2] H. Zhang, C. -X. Liu, X. -L. Qi, X. Dai, Z. Fang, and S. -C. Zhang, Nat. Phys. 5, 438442 (2009).

[3] B. A. Bernevig, T. L. Hughes, S. -C. Zhang, Science 314, 17571761 (2006).

[4] L. Fu, C. L. Kane, Phys. Rev. B 76, 045302 (2007).

[5] J. E. Moore, Nature 464, 194-198, (2010).

[6] C. L. Kane and E. J. Mele. Science 314, 16921693 (2009).

[7] D. Hsieh et al., Science 323, 919922 (2009).

[8] D. Hsieh, Y. Xia, D. Qian, L. Wray, J. H. Dil, F. Meier, J. Osterwalder, L. Patthey, J. G. Checkelsky,N. P. Ong, A. V. Fedorov, H. Lin, A. Bansil, D. Grauer, Y. S. Hor, R. J. Cava, and M. Z. Hasan, Nature 460, 11011105 (2009).

[9] S. -Y. Xu, Y. Xia, L. A. Wray, S. Jia, F. Meier, J. H. Dil, J. Osterwalder, B. Slomski, A. Bansil, H. Lin, R. J. Cava, and M. Z. Hasan, Science 332, 560564 (2011).

[10] Y. Xia, D. Qian, D. Hsieh, L Wray, A. Pal, H. Lin, A. Bansil, D. Grauer, Y. S. Hor, R. J. Cava, and M. Z. Hasan, Nat. Phys. 5, 398402 (2009).

[11] J. W. McIver, D. Hsieh, H. Steinberg, P. Jarillo-Herrero, N. Gedik, Nat. Nanotechnol. 7, 96100 (2012).

[12] C. Jozwiak, C. -H. Park, K. Gotlieb, C. Hwang, D. -H. Lee, S. G. Louie, J. D. Denlinger, C. R. Rotundu, R.J. Birgeneau, Z. Hussain, and A. Lanzara, Nat. Phys. 9, 293298 (2013).

[13] C. -H. Park, S. G. Louie, Phys. Rev. Lett. 109, 097601 (2012).

[14] C. H. Li, O. M. J. vant Erve, J. T. Robinson, Y. Liu, L. Li, B. T. Jonker, Nat. Nanotechnol. 9, 218224 (2014).

[15] S. Hong, V. Diep, S. Datta, Y. P. Chen, Phys. Rev. B 86, 085131 (2012).

[16] K. Borisov, C.-Z. Chang, J. S. Moodera, and P. Stamenov, Physical Review B 94, 094415 (2016).

[17] P. Burset, B. Lu, G. Tkachov, Y. Tanaka, E. M. Hankiewicz, and B. Trauzettel. Phys. Rev. B 92, 205424 (2015).

[18] Y. Kim, T. M. Philip, M. J. Park, and M. J. Gilbert,
Phys. Rev. B 94, 235434 (2016).

[19] L. Aggarwal, A. Gaurav, G. S. Thakur, Z. Haque, A. K. Ganguli, G. Sheet, Nature materials 15, 32-37 (2016).

[20] L. Aggarwal, S. Gayen, S. Das, R. Kumar, V. Suess, C. Felser, C. Shekhar, G. Sheet, Nature communications 8, 13974 (2017).

[21] L. Aggarwal, S. Gayen, S. Das, G. S. Thakur, A. K. Ganguli, G. Sheet, Applied Physics Letters 109, 252602 (2016).

[22] S. Das, L. Aggarwal, S. Roychowdhury, M. Aslam, S. Gayen, K. Biswas, G. Sheet, Applied Physics Letters 109, 132601 (2016).

[23] G. R. Granstrom, I. Fridman, H.-C. Lei, C. Petrovic, and J. Y. T. Wei, condmat-arXiV: 1711.00144v1 (2017).

[24] I. I. Mazin, A. A. Golubov, and B. Nadgorny, J. Appl. Phys. 89, 7576 (2001).

[25] G. J. Strijkers, Y. Ji, F. Y. Yang, C. L. Chien, and J. M. Byers, Phys. Rev. B 63, 104510 (2001).

[26] H. Zhang, C.-X. Liu, X.-L. Qi, X. Dai, Z. Fang and S.-C Zhang, Nature Physics 5, 438442 (2009).

[27] R. Vadapoo, S. Krishnan, H. Yilmaz, and C. Marin, Phys. Status Solidi B 248,3, 700705 (2011).

[28] A. Bera, K. Pal, D. V. S. Muthu, S. Sen, P. Guptasarma, U. V. Waghmare, and A. K. Sood, Physical Review Letters 110, 107401 (2013).

[29] I. Efthimiopoulos, J. Zhang, M. Kucway, C. Park, R. C. Ewing, and Y. Wang, Scientific Reports 3, 2665 (2013).

[30] W. Li, X. -Y. Wei, J. -X. Zhu, C. S. Ting, and Yan Chen, Phys. Rev. B 89, 035101 (2014).

[31] I. Efthimiopoulos, C. Buchan, and Y. Wang, Scientific Reports 6, 24246 (2016).

[32] G. H. Cao, H. J. Liu, J. H. Liang, L. Cheng, D. D. Fan, Z. Y. Zhang, arXiv:1607.05911v2.

[33] P. Cheng et al. Physical Review Letters 105, 076801 (2010).

[34] T. Hanaguri, K. Igarashi, M. Kawamura, H. Takagi, and T. Sasagawa, Physical Review B 82, 081305(R) (2010).

[35] G. E. Blonder, M. Tinkham, and T. M. Klapwijk, Phys. 
Rev. B 25, 4515 (1982).

[36] Y. G. Naidyuk, and I. K. Yanson, Point-Contact Spectroscopy ( Springer, 2005).

[37] G. Sheet , S. Mukhopadhyay, and P. Raychaudhuri , Phys. Rev. B 69, 134507 (2004).

[38] R. J. Soulen Jr., J. M. Byers, M. S. Osofsky, B. Nadgorny, T. Ambrose, S. F. Cheng, P. R. Broussard, C. T. Tanaka, J. Nowak, J. S. Moodera, A. Barry, J. M. D. Coey, Science 282, 85 (1998).

[39] P. P. Kong, F. Sun, L. Y. Xing, J. Zhu, S. J. Zhang, W.M.Li, Q. Q. Liu, X. C. Wang, S. M. Feng, X. H. Yu, J. L. Zhu, R.C.Yu, W. G. Yang, G. Y. Shen, Y. S. Zhao, R. Ahuja, H.K.Mao and C. Q. Jin. Scientific Reports 4, 6679 (2014).

[40] K. Y. Wang, K. W. Edmonds, R. P. Campion, L. X. Zhao, C. T. Foxon, and B. L. Gallagher Phys. Rev. B 72, 085201 (2005).

[41] T. McGuire and R. Potter, IEEE Transactions on Mag- netics. 11 (4): 1018 (1975).

[42] T. K. Maji1, S. K. Pal1, and D. Karmakar, AIP Con. Proc., 1942, 130053 (2018).

[43] H. Wang, H. Liu, C. -Z. Chang, H. Zuo, Y. Zhao, Y. Sun, Z. Xia, K. He, X. Ma, X. C. Xie, Q. -K. Xue, and J. Wang, Scientific Reports 4, 5817 (2014).

[44] G. Long, S. Xu, X. Cai, Z. Wu, T. Han, J. Lin, C. Cheng, Y. Cai, X. Wang and N. Wang, Nanotechnology 29, 3 (2017)

[45] F. V. Tikhonenko, A. A. Kozikov, A. K. Savchenko, and R. V. Gorbachev, Phys. Rev. Lett. 103, 226801 (2009).

[46] G. Kresse and J. Furthm uller, Phys. Rev. B 54, 11169(1996)

[47] G. Kresse, D. Joubert, Phys. Rev. B 59, 1758 (1999).

[48] A. Bansil, H. Lin and T. Das, Rev. Mod. Phys. 88, 021004 (2016). 